\section{PEER-REVIEWED ARTICLE}

Food Protection Trends, Vol 41, No. 2, p. 204-215 Copyright ${ }^{\oplus}$ 2021, International Association for Food Protection 2900 100th Street, Suite 309, Des Moines, IA 50322-3855
Mitzchie Espedido and lan Young*

School of Occupational and Public Health, Ryerson University, 350 Victoria St., Toronto, Ontario M5B 2K3, Canada

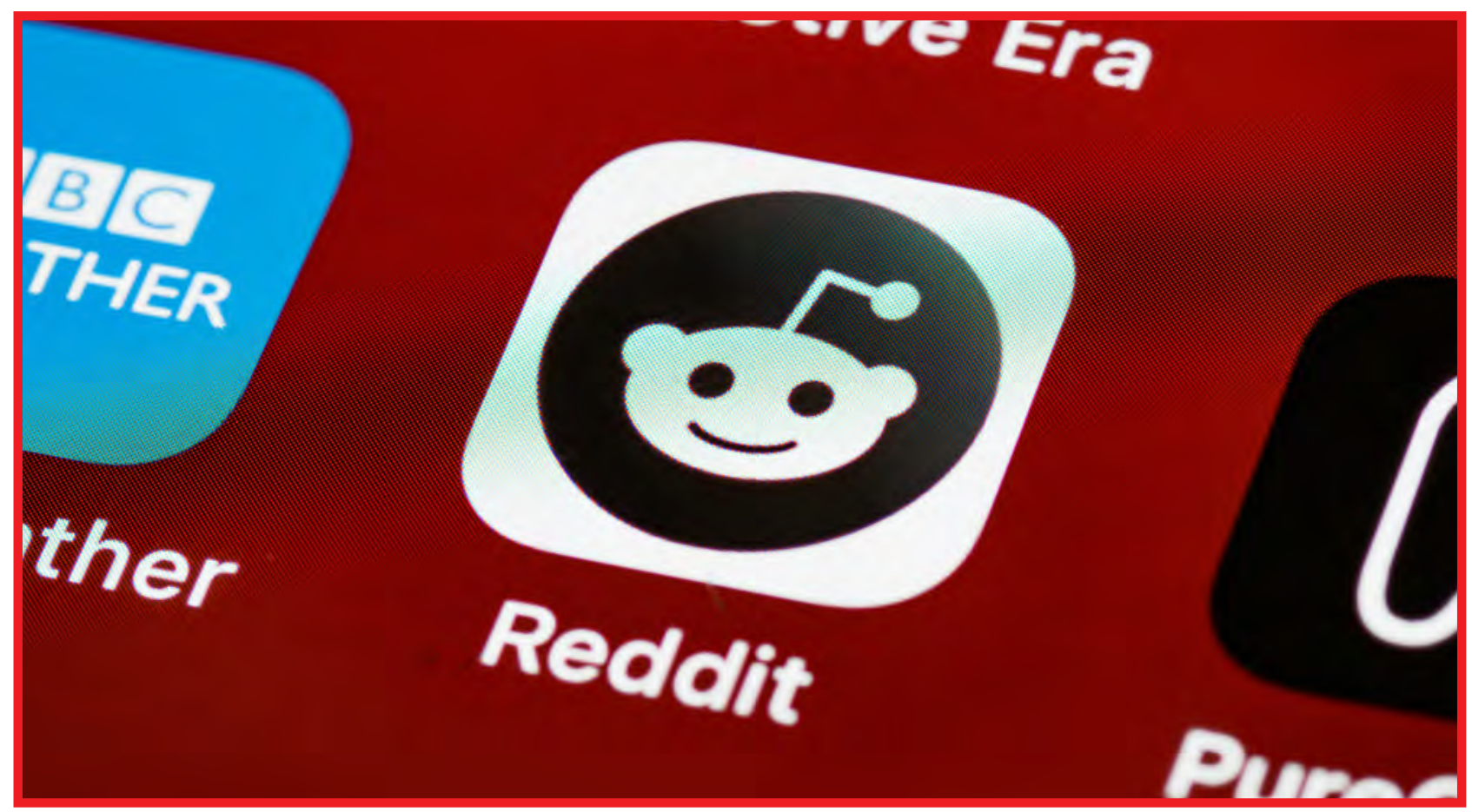

\title{
I Read It on Reddit: Food Safety Information-Seeking Preferences and Practices of Young Adults Online
}

\section{ABSTRACT}

Young adults increasingly search online for healthrelated information, including information about food safety. This cross-sectional study was conducted to evaluate the food safety information-seeking preferences and practices of young adults who use Reddit, a popular community-based website. A questionnaire was developed, guided by the technology acceptance model and theory of planned behavior, and distributed online to young adults (age 18 to 39 years) from October 2019 to January 2020. Predictors of participants' intentions to use Reddit to search for advice about safe food handling in the following 2 weeks were assessed in an ordinal regression model. Eligible responses were obtained from 209 individuals. One-third (36\%) of respondents had previously searched for food safety information on Reddit, $88 \%$ of whom adopted the advice they found. Self-reported food handling practices were generally positive, but only $50 \%$ of respondents owned a food thermometer. Respondents were more likely to intend to search for food safety advice on Reddit if they perceived the website to be useful and credible, if they previously searched Reddit for food safety information, and if they perceived safe food handling to be unpleasant. Additional education and outreach strategies targeting this website are warranted to ensure accurate food safety advice is promoted online.

\section{INTRODUCTION}

Foodborne disease has a significant burden on morbidity and mortality worldwide (15). Economic and societal impacts from health care costs, lost productivity, and other costs are also substantial (24). Sporadic cases of foodborne illness are most likely attributed to improper food preparation and handling practices in individuals' home kitchens $(21,33)$. Previous research has found that young adults do not follow several recommended safe food handling practices at home, increasing their risk of acquiring foodborne illness $(5,6,34)$.

Young adults frequently use online communities such as Reddit to search and browse for health-related information (31). However, health information and advice disseminated in online communities may contain misinformation and 
inaccuracies $(9,35)$. For example, a recent study analyzed the accuracy and reliability of food safety information posted by users on Reddit and other online, communitybased question-and-answer platforms, finding that the advice given is widely inconsistent in comparison to government recommendations for safe food handling (35). Prior research shows that consumer safe food-handling practices at home are often influenced by their friends, families, and social networks and that consumers are increasingly using online resources to access information about food safety $(11,22,37,38)$.

Reddit is an community-based website that was ranked as the sixth most popular website in the United States in 2020 (3). Reddit allows users to post original content (e.g., questions) or links to other articles (e.g., news), which are then commented on by other users in the community (31). Users can then upvote or downvote each response or comment in a post, with those receiving more upvotes rising to the top of the page. An account is not required to browse and review posts on the website, but one is needed to post content or to comment on other users' posts. Reddit contains many topic-specific communities called subreddits, in which users can post and discuss more specific issues, and each subreddit is managed by a team of volunteer moderators who ensure quality control (e.g., by removing irrelevant, insincere, or low-quality posts and comments).

Theories of behavior change can be used to identify and explain the key factors that influence consumers' safe food handling behaviors $(23,25,26,36)$. The theory of planned behavior (TPB) is one of the most commonly applied theories in this area $(23,25,26,36)$. TPB states that an individual's attitude toward a behavior, their subjective norm (i.e., perceived influence of other people important to them, such as friends and family), and their perceived behavioral control (i.e., self-confidence and control over the behavior) all influence their intention to change a behavior (2). Behavior change is then influenced directly by an individual's intentions and their perceived behavioral control (2). Another theory, called the technology acceptance model (TAM), can be used to explain people's intentions to use websites to search for health-related information (39). TAM suggests that an individual's intentions to adopt or use a new technology are primarily influenced by the perceived ease of use of the technology and its perceived usefulness (10). Yun and Park (40) applied this model to online health information-seeking behavior, adding the construct of perceived credibility of the website as another important determinant of intentions.

There is a need to investigate the extent to which young adults who use online question-and-answer communities, such as Reddit, search for and use food safety information provided by their peers to inform their food safety practices at home. Therefore, a study was conducted, informed by TPB and TAM, to survey users of Reddit about their food safety information-seeking intentions and to determine factors associated with this outcome. We selected Reddit for this study given its wide popularity among young adults and because previous research has found that it is frequently used for peer dissemination of information and advice about food safety $(3,31,35)$. The study results can be used to inform food safety professionals about possible online communication strategies and considerations for young adults.

\section{MATERIALS AND METHODS Study design}

A cross-sectional study was conducted from October 2019 to January 2020 to evaluate the food safety information-seeking preferences and practices of young adults who use the Reddit website. The target population for the survey was young adults (age 18 to 39 years) who use Reddit at least occasionally. In addition, participants were required to be responsible for preparing meals at home at least once a week. There were no location-based or other exclusion criteria for the survey. The study was reviewed and approved by the Ryerson University Research Ethics Board (REB No. 2019-381).

\section{Participant selection}

We distributed a link to the questionnaire on the following Reddit communities: $r$ /SampleSize, $r$ /foodsafety, $r$ / Cooking, $r$ /cookingforbeginners, and $r /$ AskCulinary. The $\mathrm{r}$ /SampleSize community is designed for the exchange of research and other surveys, whereas the other subreddits are topic-specific communities that include discussion and questions related to cooking at home, including food handling and safety (35). The moderators of some communities ( $r$ /Cooking, $r$ /cookingforbeginners, and $r$ / AskCulinary) occasionally removed the survey link despite there being no explicit rules about not posting surveys in these subreddits. Because of a low initial response, we expanded the survey link distribution in November 2019 to four public Facebook groups designed for sharing of research surveys: The Research Survey Exchange Group, Thesis/ Survey Questionnaire Filling Group, Survey Exchange, and Survey Sharing 2019. Survey links were reposted in approximately 2 -week intervals during the study period.

\section{Questionnaire design and questions}

A questionnaire was developed based on the TAM and TPB frameworks, with specific questions adapted from previous studies and questionnaires in the literature (10, $13,25,31,39)$. The online questionnaire was hosted on the Opinio survey platform at Ryerson University. It contained 30 questions, including 3 screening questions for eligibility. The first section contained 6 questions about participants' Reddit use: hours of use per day (number of hours); primary use of Reddit (active poster or commenter versus passive 
reviewer of information); prior searching for food safety information on Reddit (yes versus no) and, if yes, type of food safety information searched for (handwashing, washing of foods, cleaning and sanitation, food storage and thawing, cooking, safe foods, and other) and whether the information was adopted at home (yes versus no); and intention to search for advice on Reddit about food safety practices at home in the following 2 weeks (7-point scale from very unlikely to very likely).

The next section asked about TAM and TPB constructs, all of which were measured on a 7-point scale. Three TAM constructs were investigated: perceived usefulness of Reddit, perceived ease of use of Reddit, and perceived credibility of information on Reddit $(10,39)$. Perceived usefulness (I find that searching for information on Reddit is useful: strongly disagree to strongly agree) and ease of use (I find that searching for information on Reddit is very easy to very difficult) were measured with one question item each. Perceived credibility of information was measured with three items (In general, I find that information posted by users on Reddit is very inaccurate to very accurate, very incredible to very credible, and very unbelievable to very believable). TPB constructs of attitudes, subjective norm, and perceived behavioral control were measured with two question items each. The attitude questions (For me, preparing, handling, and storing food safely at home is ... ) included one item for instrumental attitudes (very unnecessary to very necessary) and another for experiential attitudes (very unpleasant to very pleasant). Subjective norm included one question item for injunctive norm (Most people who are important to me think that I should prepare, handle, and store food safely at home: strongly disagree to strongly agree) and one for social pressure (I feel under social pressure, e.g., from family, friends, or society at large, to prepare, handle, and store food safely at home: strongly disagree to strongly agree). Perceived behavioral control included one question item for selfefficacy (I am confident that if I wanted to, I could prepare, handle, and store food safely at home: strongly disagree to strongly agree) and one for controllability (Preparing, handling, and storing food safely at home is beyond my control: strongly disagree to strongly agree).

The next seven questions asked participants about their self-reported safe food handling practices at home, including handwashing frequency (never to always), food thermometer ownership (yes versus no) and frequency of use (never to always), methods of checking the degree of cooking doneness (visual, time, taste, touch, temperature, and other), frequency of washing of poultry before cooking (never to always), cooling time for leftovers $(2 \mathrm{~h}$ or less, 2 to $4 \mathrm{~h}, 4$ to $6 \mathrm{~h}$, or $>6 \mathrm{~h}$ ), and storage times for leftovers ( 1 day or less, 2 days, 3 days, 4 days, or $>4$ days). The "never to always" response options were measured on a 5-point scale. Sociodemographic information was collected on country of residence (United States, United Kingdom, Canada, or other), gender (male, female, or nonbinary), age group ( 18 to 24,25 to 32 , or 33 to 39), education level (less than high school, high school or equivalent, apprenticeship or trades, some college or university, college or university degree, postgraduate degree, or other), prior foodborne illness in past year (yes versus no), experience in the food service industry (yes versus no), and main source of information about food safety at home (family and friends, experience, recipe blogs, Reddit, other websites, social media, health professionals, cookbooks, or other).

The questionnaire was pretested through a cognitive interviewing process (30). Ten young adults meeting the eligibility criteria were recruited for in-person cognitive interviews. Each question was read to the participants, and they were asked to communicate their response and thought process in a think-aloud approach. Feedback from this process resulted in removing and rewording some questions to improve the clarity and conciseness of the questionnaire before implementation.

\section{Data analysis}

Responses were downloaded to Microsoft Excel 2019 (Microsoft Corporation, Redmond, WA) spreadsheets for formatting and transferred to Stata 14 IC (StataCorp, College Station, TX) for analysis. Descriptive tabulations and measures of central tendency were calculated for all questions. Fisher's exact test was conducted to explore associations between respondents' history of searching for food safety information on Reddit and their self-reported safe food handling practices; for these tests, a Bonferroni-adjusted $P$-value of $<0.008$ was used to indicate statistical significance ( 0.05 of 6 comparisons). Theoretical constructs were reverse coded as necessary so that higher values corresponded with positive outcomes. Mean composite variables were constructed for all theory-based constructs if Cronbach's alpha was $\geq 0.70$. Only the TAM credibility construct was sufficiently reliable; for TPB constructs, individual question items were used instead of composite constructs. Pairwise Spearman correlations were conducted between each of these variables to ensure that none were highly correlated $(r \geq 0.8)$.

An ordinal regression model was constructed to identify predictors of participants' intentions to use Reddit in the following 2 weeks to search for advice about safe food handling at home. The outcome was a 7-point ordinal response (very unlikely to very likely). The following variables were evaluated as potential predictors of this outcome: age group ( 18 to 24,25 to 32 , or 33 to 39 ), gender (male versus female), country (United States, United Kingdom, Canada, or other), education (high school, trades, or other; some college or university; bachelor's degree; or postgraduate degree), experienced domestic foodborne illness in past year (yes versus no), food service industry experience (yes versus no), frequency of using Reddit (daily versus less often), primary use of Reddit (active versus passive), history of searching for food safety information 
on Reddit (yes versus no), and nine theoretical constructs each evaluated as a continuous measure on a 7-point scale (Reddit usefulness, usability, and credibility and safe food handling instrumental and experiential attitudes, self-efficacy, controllability, injunctive subjective norm, and social pressure).

Each variable was first screened in univariable models. If significant at a $P \leq 0.20$ level, variables were then evaluated in a multivariable model. Variables were removed from the multivariable model if their $95 \%$ confidence intervals crossed the null or if their removal changed the coefficients of other variables by $>20 \%$. The proportional odds assumption of the model was assessed with a Brandt test, with $P<0.05$ indicating violation of the assumption (1). This assumption assumes that the relationship between predictors and outcome is the same for each combination of outcome categories, so only one set of coefficients is estimated for each predictor in the model (1).

\section{RESULTS}

\section{Respondent characteristics, Reddit use, and self- reported practices}

A total of 308 individuals started the survey, and 234 individuals completed it. Of these, 25 responses were $>50 \%$ blank and removed from analysis, leaving 209 usable responses. The sociodemographic and Reddit use characteristics of respondents are noted in Table 1. Most respondents were age 18 to 24 years (44\%), female (67\%), located in the United States (50\%), and enrolled in or had completed a bachelor's or postgraduate degree (84\%). Most respondents primarily obtained food safety information from their family and friends (28\%), websites $(27 \%)$, or self experience (22\%). Most respondents (73\%) indicated that they use Reddit daily, and among these respondents, the median reported time of daily use was $2 \mathrm{~h}$ (interquartile range $=1$ to $2.75 \mathrm{~h}$ ). The primary use of Reddit was to passively review information (75\%). Approximately onethird (36\%) of respondents previously searched for food safety information on Reddit, examining a variety of food safety topics. Among these respondents, $88 \%$ reported adopting the information obtained from Reddit to inform their food handling practices at home.

The self-reported safe food handling practices of respondents are shown in Table 2. Most respondents (82\%) reported always or often washing their hands before food preparation, whereas only $50 \%$ reported owning a food thermometer. Among those who reported consuming meat or poultry $(n=181)$, the most common method of determining the degree of cooking doneness was visual methods $(81 \%)$, whereas most respondents $(71 \%)$ reported rarely or never washing poultry before cooking it. Among respondents who owned a food thermometer and prepared meat or poultry meals, $58 \%$ reported always or often using a thermometer to check doneness. Most respondents
(76\%) reported refrigerating leftovers within $2 \mathrm{~h}$, and $86 \%$ reported consuming meat or poultry leftovers within 4 days. Respondents who had previously searched for food safety information on Reddit had safe food handling behaviors similar to those who did not previously search for food safety information on this website (Table 3).

Summary information for the theoretical construct variables is shown in Table 4. In general, participants had slightly above-average perceptions of the usefulness, ease of use, and credibility of Reddit (Table 4). Participants had positive attitudes and strongly perceived behavioral control and injunctive subjective norm toward safe food handling at home; however, they reported low perceived social pressure related to safe food handling (Table 4). Respondents had low to moderate intentions to use Reddit over the following 2 weeks to search for food safety information (mean $=3.03$, standard deviation $=1.88$, range $=1$ to 7 ).

\section{Predictors of intentions to use Reddit to search for food safety information}

The final multivariable modeling results are shown in (Table 5). There were no issues with the proportional odds assumption $(P=0.226)$. Those who perceived Reddit to be a useful and credible source of information and those who reported previously searching for food safety information on Reddit were more likely to intend to search for food safety information on Reddit in the following 2 weeks (Table 5). Those who had a higher experiential attitude toward safe food handling at home (i.e., perceived it to be a pleasant experience) were less likely to intend to search Reddit for food safety information over the following 2 weeks, though this variable had a confidence interval that included the null (Table 5).

\section{DISCUSSION}

The study sample was targeted toward young adults who use Reddit. Respondent demographics (e.g., gender distribution and food service industry experience) were similar to those in prior surveys of young adults and university or college students $(7,12,14)$. Most respondents in this study used Reddit at least once per day and indicated that they primarily review content rather than actively post or comment on others' posts, which is also similar to a prior study of Reddit users (31). Although it was not their main source of obtaining food safety information, one-third of respondents indicated that they previously searched for food safety information on Reddit, and the reported rate of adoption of this advice was high (88\%). This finding suggests a high level of trust in the food safety information shared on this platform and corresponds with research that shows that consumers' safe food-handling practices and informationseeking behaviors are often influenced by their peers and other sources they trust $(22,29,38)$.

Most self-reported safe food-handling behaviors of respondents (e.g., handwashing and food cooling and storage 


\section{TABLE 1. Sociodemographic characteristics and food safety information-seeking behaviors}

among a sample of 209 Reddit users, 2019-2020

Characteristic

n $(\%)$

Age $(\mathrm{n}=209)$

$\begin{array}{r}\hline 18-24 \\ \hline 25-32 \\ \hline 33-39\end{array}$

$92(44.0)$

$75(35.9)$

$42(20.1)$

Gender $(\mathrm{n}=207)$

Female

Male

Non-binary

Country of residence $(n=201)$

\begin{tabular}{l|c}
\hline U.S. & $100(49.8)$ \\
\hline UK & $26(12.9)$ \\
\hline Canada & $23(11.4)$ \\
\hline Other & $52(25.9)$ \\
\hline Education $(\mathbf{n}=\mathbf{2 0 7})$ &
\end{tabular}

Education $(\mathrm{n}=207)$

High school, trades, apprenticeship, or other

$34(16.4)$

Some college or university

$49(23.7)$

College or university bachelor's degree

Post-graduate degree

$83(40.1)$

$41(19.8)$

Experienced domestically acquired foodborne illness in past year $(n=203)$

\begin{tabular}{|c|c|}
\hline Yes & $42(20.7)$ \\
\hline No & $161(79.3)$ \\
\hline \multicolumn{2}{|c|}{ Current or previous work in the food service industry $(n=205)$} \\
\hline Yes & $115(56.1)$ \\
\hline No & $90(43.9)$ \\
\hline \multicolumn{2}{|c|}{ Main source of food safety information at home $(n=205)$} \\
\hline Family and friends & $58(28.3)$ \\
\hline Websites on the Internet (other than Reddit) & $56(27.3)$ \\
\hline Self-experience & $44(21.5)$ \\
\hline Food safety courses/certification & $18(8.8)$ \\
\hline Health professionals & $13(6.3)$ \\
\hline Social media (including Reddit) & $7(3.5)$ \\
\hline Cookbooks and cooking/TV shows & $6(3.0)$ \\
\hline Recipe blogs & $5(2.4)$ \\
\hline Other (unspecified) & $1(0.5)$ \\
\hline \multicolumn{2}{|l|}{ Frequency of using Reddit $(n=209)$} \\
\hline Daily & $153(73.2)$ \\
\hline Less than once per day & $56(26.8)$ \\
\hline \multicolumn{2}{|l|}{ Primary use of Reddit $(n=205)$} \\
\hline Passively review information & $154(75.1)$ \\
\hline Actively participate in comments and posts & $51(24.9)$ \\
\hline
\end{tabular}

Actively participate in comments and posts 
TABLE 1. Sociodemographic characteristics and food safety information-seeking behaviors among a sample of 209 Reddit users, $2019-2020$ (cont.)

Characteristic

n $(\%)$

Previously searched for food safety information on Reddit? $(n=209)$

\begin{tabular}{l|c}
\hline Yes & $75(36.1)$ \\
\hline No & $134(64.1)$ \\
\hline Types of food safety-related information searched for on Reddit $(\mathbf{n}=\mathbf{7 5})^{\mathrm{a}, \mathrm{b}}$ & $50(66.7)$ \\
\hline Safe chilling, thawing, or storage of foods & $40(53.3)$ \\
\hline Safe cooking of foods & $31(41.3)$ \\
\hline Determining what foods are safe to eat & $16(21.3)$ \\
\hline Washing or rinsing of foods & $12(16.0)$ \\
\hline Cleaning, sanitation, or washing of utensils or surfaces & $4(5.3)$ \\
\hline Food fermentation or canning & $4(5.3)$ \\
\hline Handwashing & \\
\hline Adopted food safety information from Reddit in at-home practices $(\mathbf{n}=74)^{\mathrm{a}}$ & $65(87.8)$ \\
\hline Yes & $9(12.2)$ \\
\hline No & \\
\hline
\end{tabular}

${ }^{a}$ These questions were only tabulated for those who indicated that they had previously searched for food safety information on Reddit $(\mathrm{n}=75)$.

${ }^{b}$ Multiple selections were possible for this question (i.e., percentages do not add to $100 \%$ ).

times) were consistent with recommended food safety practices and prior population-based consumer surveys ( 7 , $17,18,20,28,32)$. However, only half of the respondents reported owning a food thermometer, lower than other surveys of the general population that found approximately two-thirds of consumers own a thermometer $(20,32)$. This corresponds with prior research indicating younger adults are less likely to own a food thermometer than older adults (18, 19). Most respondents who reported owning a thermometer indicated they used it often or always to check the degree of cooking doneness, but improvements could be made in this area, because the most common method of checking doneness among respondents was through visual methods (e.g., meat color or juices run clear) followed by cooking time. This is a concern, because sensory methods are not reliable to confirm the destruction of microbial pathogens on meat (4). Additional online outreach is needed with younger adults to increase their access to and use of a food thermometer to check cooking doneness. Consumers with lower incomes are less likely to own a food thermometer (19), which likely includes many college and university students and recent graduates that comprise the young adult demographic. Programs should be considered that provide free or low-cost food thermometers to this population group to promote their use.
The most surprising study finding was that most survey respondents reported rarely or never washing poultry before cooking it, which is opposite to prior surveys (16, $20,32)$. This finding may reflect a combination of factors, including that younger adults are less likely to wash poultry before cooking than older adults (18), that food safety education campaigns (e.g., "Don't Wash Your Chicken!") and recommendations aimed at reducing consumer use of this behavior may have been effectively disseminated to and reached this population online (15), and that our sample may have included a higher proportion of food safety experts than other consumer surveys. Washing or rinsing of poultry is not recommended by public health and food safety authorities, because it can transfer bacteria to the kitchen sink, which can lead to cross-contamination of ready-to-eat foods via consumers' hands or utensils (8). Further research is needed to investigate information sources and influences of this behavior among younger versus older adults to inform possible strategies to educate those who continue to engage in this risky behavior.

A strong association was found between respondents' prior searching behavior and their future intentions to search for food safety information on Reddit. This result was not surprising and corresponds with another study that found a strong association between the information-seeking 


\section{TABLE 2. Self-reported safe food-handling practices among a sample of 209 Reddit}

users, $2019-2020$

Characteristic

n $(\%)$

Frequency of handwashing before preparing food $(n=208)$

\begin{tabular}{l|c}
\hline Always & $109(52.4)$ \\
\hline Often & $61(29.3)$ \\
\hline Sometimes & $28(13.5)$ \\
\hline Rarely & $10(4.8)$ \\
\hline Never & $0(0)$ \\
\hline
\end{tabular}

Prepare meals at home that contain meat or poultry $(n=208)$

\begin{tabular}{l|c}
\hline Yes & $181(87.0)$ \\
\hline No & $27(13.0)$ \\
\hline
\end{tabular}

Methods used to determine cooking doneness of meat or poultry $(n=181)^{\mathrm{a}, \mathrm{b}}$

\begin{tabular}{l|c}
\hline Visual & $147(81.2)$ \\
\hline Time & $104(57.5)$ \\
\hline Internal temperature & $92(50.8)$ \\
\hline Touch or texture & $79(43.7)$ \\
\hline Taste & $20(11.1)$ \\
\hline Own a food thermometer $(\mathbf{n}=\mathbf{2 0 8})$ & $107(51.4)$ \\
\hline Yes & $101(48.6)$ \\
\hline No & \\
\hline
\end{tabular}

Frequency of using a food thermometer to check cooking doneness of meat and poultry $(n=104)^{\mathrm{a}, \mathrm{c}}$

\begin{tabular}{l|c}
\hline Always & $31(29.8)$ \\
\hline Often & $29(27.9)$ \\
\hline Sometimes & $26(25.0)$ \\
\hline Rarely & $15(14.4)$ \\
\hline Never & $3(2.9)$ \\
\hline Wash or rinse poultry before cooking it $\left(\mathbf{n}=\mathbf{1 8 0 ) ^ { \mathrm { a } }}\right.$ &
\end{tabular}

Wash or rinse poultry before cooking it $(n=180)^{\mathrm{a}}$

\begin{tabular}{l|c}
\hline Always & $27(15.0)$ \\
\hline Often & $12(6.7)$ \\
\hline Sometimes & $13(7.2)$ \\
\hline Rarely & $26(14.4)$ \\
\hline Never & $102(56.7)$ \\
\hline Usual cooling time at room temperature for leftovers $(\mathbf{n}=\mathbf{2 0 1})$ & $153(76.1)$ \\
\hline 2h or less & $38(18.9)$ \\
\hline More than 2-4h & $5(2.5)$ \\
\hline More than 4-6h & $5(2.5)$ \\
\hline More than 6 $\mathrm{h}$ & \\
\hline Keep or store leftovers containing meat or poultry at home $(\mathbf{n}=\mathbf{1 8 0})^{\mathrm{a}}$ & $176(97.8)$ \\
\hline Yes & $4(2.2)$ \\
\hline No & \\
\hline
\end{tabular}




\section{TABLE 2. Self-reported safe food-handling practices among a sample of 209 Reddit}

users, 2019-2020 [cont.]

\begin{tabular}{l|c} 
Characteristic & $\mathbf{n}(\%)$ \\
\hline Usual number of days that leftovers containing meat or poultry are kept before consumption $(\mathbf{n}=\mathbf{2 0 1})^{\mathrm{a}, \mathrm{d}}$ & \multicolumn{1}{|c}{} \\
\hline 1 day or less & $29(16.5)$ \\
\hline 2 days & $48(27.3)$ \\
\hline 3 days & $49(27.8)$ \\
\hline 4 days & $25(14.2)$ \\
\hline 5 days or longer & $25(14.2)$ \\
\hline \multicolumn{2}{|c}{ These questions were tabulated only for respondents that indicated that they prepare meals at home containing meat or poultry $(\mathrm{n}=181)}$. \\
'Multiple selections were possible for this question (i.e., percentages do not add to 100\%). \\
$\begin{array}{l}\text { 'This question was only tabulated for respondents that indicated they own a food thermometer }(\mathrm{n}=107) . \\
\text { dThis question was only tabulated for respondents that indicated they keep or store leftovers containing meat or poultry }(\mathrm{n}=176) .\end{array}$
\end{tabular}

behavior and adoption of health-related advice among users of this website (31). This finding suggests that those who have already looked to Reddit for advice about safe food handling are likely to do so again. Furthermore, there were no differences in self-reported safe food handling practices among those who previously searched for food safety information on Reddit and those who did not, indicating that behaviors are largely consistent between these groups of respondents. This finding suggests that some positive food safety messages are likely being widely disseminated among the Reddit community, such as not washing poultry before cooking, but efforts are still needed to improve other areas, particularity food thermometer ownership and use.

Respondents who perceived Reddit to be a useful and credible source of information were more likely to intend to search for food safety information on this platform in the following 2 weeks. This result corresponds with a prior study that found these TAM constructs predicted consumers' intention to use health-related information on the Internet (39) and with a study that found consumers strongly value the accuracy and trustworthiness of online information sources about foodborne illness (22). Each Reddit community has a team of volunteer moderators who help to remove spam and poor-quality answers, and answers will move toward the bottom of the list of responses, or even be hidden, if they receive enough downvotes from other users. In addition to trust in their peers $(29,38)$, it is possible that the survey respondents viewed these mechanisms as important quality-control strategies, enhancing their perceived credibility of the information provided. This is supported by some evidence, because a prior study of Reddit and other community-based question-and-answer websites found that top-rated answers to food safety questions were more likely to be correct than other answers (35). However, much of the food safety advice provided on these websites contains inaccuracies (35), indicating that additional efforts are needed to enhance reliable food safety messaging in online communities.

Respondents who viewed safe food handling at home as a pleasant activity were less likely to intend to search for food safety information on Reddit in the following 2 weeks, which suggests that those who enjoy safe food handling may not require additional information to inform their behaviors. Previous research has shown that emotions may be an important factor influencing consumers' safe food-handling decisions (27) and could be integrated into future outreach and education strategies. None of the other TPB constructs were related to respondents' food safety searching intentions on Reddit, indicating that these intentions are driven more by consumers' perceptions of and prior use of the website than their safe food-handling perceptions and beliefs.

There are several limitations to this study. The survey was distributed to targeted Reddit and Facebook communities designed for exchange of surveys and focused on cooking and food handling at home. Therefore, the respondents may not be representative of the broader population of young adults or Reddit users. Additional research is warranted to investigate the food safety intentions and behaviors of young adults who use other popular community-based websites and social media platforms. Only two-thirds of those who started filling out the survey completed it and were included in the analysis. Future studies in this area should consider the use of an incentive to increase response and completion rates. Despite these limitations, our final sample size was comparable to that of prior studies in this area $(23,25,26,39)$ and was likely sufficient to estimate the predictive model under investigation in this study (13). 


\section{TABLE 3. Association between searching for food safety information and self-reported safe}

food-handling behaviors among a sample of 209 Reddit users, 2019-2020

\begin{tabular}{|c|c|c|c|}
\hline \multirow[b]{2}{*}{ Behavior } & \multicolumn{3}{|c|}{$\begin{array}{l}\text { Previously searched for food } \\
\text { safety information on Reddit }\end{array}$} \\
\hline & Yes $(\%)$ & No $(\%)$ & $P$-value \\
\hline Frequency of handwashing before preparing food & & & 0.185 \\
\hline Always & $43(58.1)$ & $66(49.3)$ & \\
\hline Often & $22(29.7)$ & $39(29.1)$ & \\
\hline Sometimes & $5(6.8)$ & $23(17.2)$ & \\
\hline Rarely & $4(5.4)$ & $6(4.5)$ & \\
\hline Never & $0(0)$ & $0(0)$ & \\
\hline Own a food thermometer & & & 0.192 \\
\hline Yes & $43(58.1)$ & $64(47.8)$ & \\
\hline No & $31(41.9)$ & $70(52.2)$ & \\
\hline Frequency of using a food thermometer to check cooking doneness of meat and poultry $(\mathbf{n}=104)^{\mathrm{a}, \mathrm{b}}$ & & & 0.353 \\
\hline Always & $13(30.2)$ & $18(29.5)$ & \\
\hline Often & $10(23.3)$ & $19(31.2)$ & \\
\hline Sometimes & $11(25.6)$ & $15(24.6)$ & \\
\hline Rarely & $9(20.9)$ & $6(9.8)$ & \\
\hline Never & $0(0)$ & $3(4.9)$ & \\
\hline Wash or rinse poultry before cooking it $(\mathrm{n}=180)^{\mathrm{a}}$ & & & 0.742 \\
\hline Always & $8(11.9)$ & $19(16.8)$ & \\
\hline Often & $4(6.0)$ & $8(7.1)$ & \\
\hline Sometimes & $6(9.0)$ & $7(6.2)$ & \\
\hline Rarely & $8(11.9)$ & $18(15.9)$ & \\
\hline Never & $41(61.2)$ & $61(54.0)$ & \\
\hline Usual cooling time at room temperature for leftovers & & & 0.818 \\
\hline 2 hours or less & $53(74.7)$ & $100(76.9)$ & \\
\hline More than 2 hours to 4 hours & $15(21.1)$ & $23(17.7)$ & \\
\hline More than 4 hours to 6 hours & $2(2.5)$ & $3(2.3)$ & \\
\hline More than 6 hours & $1(1.4)$ & $4(3.1)$ & \\
\hline Usual number of days that leftovers containing meat or poultry are kept before consumption ${ }^{\mathrm{a}, \mathrm{c}}$ & & & 0.171 \\
\hline 1 day or less & $11(16.4)$ & $18(16.5)$ & \\
\hline 2 days & $16(23.9)$ & $32(29.4)$ & \\
\hline 3 days & $18(26.9)$ & $31(28.4)$ & \\
\hline 4 days & $15(22.4)$ & $10(9.2)$ & \\
\hline 5 days or longer & $7(10.5)$ & $18(16.5)$ & \\
\hline
\end{tabular}

${ }^{a}$ These questions were tabulated only for respondents that indicated that they prepare meals at home containing meat or poultry $(\mathrm{n}=181$ ).

'This question was only tabulated for respondents that indicated they own a food thermometer $(\mathrm{n}=107)$.

'This question was only tabulated for respondents that indicated they keep or store leftovers containing meat or poultry $(n=176)$. 
TABLE 4. Psychosocial constructs related to perceptions of Reddit and safe food handling at home among a sample of 209 Reddit users, $2019-2020$

\begin{tabular}{|c|c|c|c|c|c|}
\hline Theory / construct ${ }^{a}$ & $\mathbf{N}$ & Mean & SD & Items & Cronbach's alpha \\
\hline \multicolumn{6}{|l|}{ TAM - perceptions of Reddit } \\
\hline Perceived usefulness & 205 & 5.01 & 1.60 & 1 & $\mathrm{~N} / \mathrm{A}$ \\
\hline Perceived ease of use & 208 & 4.60 & 1.60 & 1 & $\mathrm{~N} / \mathrm{A}$ \\
\hline Perceived credibility of information & 209 & 4.43 & 1.00 & 3 & 0.80 \\
\hline \multicolumn{6}{|l|}{ TPB - safe food handling at home } \\
\hline Attitudes - instrumental & 208 & 6.16 & 1.15 & 1 & $\mathrm{~N} / \mathrm{A}$ \\
\hline Attitudes - experiential & 207 & 5.08 & 1.46 & 1 & $\mathrm{~N} / \mathrm{A}$ \\
\hline Subjective norm - injunctive & 207 & 5.73 & 1.45 & 1 & $\mathrm{~N} / \mathrm{A}$ \\
\hline Subjective norm - social pressure & 209 & 3.47 & 1.97 & 1 & $\mathrm{~N} / \mathrm{A}$ \\
\hline Perceived behavioral control - self-efficacy & 209 & 6.39 & 0.94 & 1 & $\mathrm{~N} / \mathrm{A}$ \\
\hline Perceived behavioral control - controllability & 209 & 6.27 & 1.18 & 1 & $\mathrm{~N} / \mathrm{A}$ \\
\hline
\end{tabular}

aThe response range for all variables was 1 to 7 . The sample size varies by construct because some participants had missing information for some variables.

bNA, not applicable.

TABLE 5. Multivariable ordinal regression model of determinants of respondents intentions to search for food safety advice on Reddit in the following two weeks

\begin{tabular}{|c|c|c|c|c|}
\hline Predictor ${ }^{\mathrm{a}}$ & $\mathrm{OR}^{\mathrm{b}}$ & SE & $95 \% \mathrm{CI}$ & $P$-value ${ }^{c}$ \\
\hline Perceived usefulness of Reddit & 1.60 & 0.16 & $1.32,1.94$ & $<0.001$ \\
\hline Perceived credibility of Reddit & 1.38 & 0.21 & $1.03,1.84$ & 0.031 \\
\hline Experiential attitude toward safe food handling at home & 0.83 & 0.08 & $0.69,1.00$ & 0.051 \\
\hline Previously searched for food safety information on Reddit (yes vs. no) & 8.46 & 2.60 & $4.70,15.23$ & $<0.001$ \\
\hline
\end{tabular}

$\mathrm{CI}=95 \%$ confidence intervals $; \mathrm{OR}=$ odds ratio; $\mathrm{SE}=$ standard errors.

${ }^{a}$ The Brandt test for the proportional odds assumption was not significant $(P=0.226)$. The pseudo- $\mathrm{R}^{2}$ for the model was 0.145 .

The model sample size was 203 due to some participants having missing information for some variables.

${ }^{\mathrm{b}} \mathrm{CI}$, 95\% Confidence interval; OR, odds ratio.

${ }^{c} P$-values for the significance of each variable were obtained from likelihood ratio tests.

The moderators in the cooking subreddits occasionally removed our recruitment link, which could have reduced exposure to consumers in those communities. Moreover, the inclusion of the food safety subreddit may have resulted in an overrepresentation of food safety experts in our sample, which could have contributed to the relatively high self-reported use of safe food handling behaviors among respondents. Similar to other surveys that rely on selfreporting, the behaviors reported among respondents could have been influenced by social desirability bias and may be overestimated. Although we pretested the questionnaire using a cognitive interviewing approach, the lack of reliability in TPB constructs suggests that additional pretesting (e.g., a small pilot survey) could have been useful for those variables. Despite these limitations, we believe the sample provides an informative cross-section of the food safety and informationseeking preferences, intentions, and behaviors of young adults who use Reddit.

The results of the study indicate that young adults who searched for food safety information on Reddit are likely to adopt the advice they obtained from other Reddit users. The self-reported safe food handling behaviors of respondents were largely consistent with recommended guidelines. Behavioral gaps were identified in thermometer ownership 
and the use of visual methods to check for meat cooking doneness. Future research and education are needed to improve thermometer ownership and use among young adults. Respondents viewed Reddit to be a trusted and useful source of information, and these perceptions informed their intention to use the website as a source of food safety information. Therefore, targeted food safety education and outreach efforts should be conducted on Reddit and similar websites to promote and encourage safe food handling at home.

\section{ACKNOWLEDGMENTS}

The authors thank all study participants for completing the survey, as well as those who participated in the cognitive interviewing pretest. Salary support for the primary author was made possible through Ryerson University's Work Study Research Assistant program.

\section{REFERENCES}

1. Agresti, A. 2010. Analysis of ordinal categorical data. John Wiley \& Sons, Inc., Hoboken, NJ.

2. Ajzen, I. 1991. The theory of planned behavior. Organ. Behav. Hum. Decis. Process. 50:179-211.

3. Alexa Internet Inc. 2020. Top sites in the United States. Available at: https://www. alexa. com/topsites/countries/US. Accessed 24 June 2020

4. Boqvist, S., L.-L. Fernström, B. W. Alsanius, and R. Lindquist. 2015. Escherichia coli O157:H7 reduction in hamburgers with regard to premature browning of minced beef, colour score and method for determining doneness. Int. J. Food Microbiol. 215:109-116.

5. Byrd-Bredbenner, C., J. M. Abbot, V. Wheatley, D. Schaffner, C. Bruhn, and L. Blalock. 2008. Risky eating behaviors of young adults-implications for food safety education. J. Am. Diet. Assoc. 108:549-552.

6. Byrd-Bredbenner, C., J. Maurer, V. Wheatley, E. Cottone, and M. Clancy. 2007. Food safety hazards lurk in the kitchens of young adults. J. Food Prot. 70:991-996.

7. Byrd-Bredbenner, C., J. Maurer, V. Wheatley, D. Schaffner, C. Bruhn, and L. Blalock. 2007. Food safety self-reported behaviors and cognitions of young adults: results of a national study. J. Food Prot. 70:1917-1926.

8. Cates, S. C., E. Shumaker, B. Chapman, L. Shelley, R. M. Goulter, M. Kirchner, L. Goodson, and L.-A. Jaykus. 2019. Food safety consumer research project: meal preparation experiment related to poultry washing. Available at: https://www.fsis. usda.gov/wps/wcm/connect/9bb3a252-e12e40e5-b76b-cb46a2322c3f/FSCRP Year+2 Final_Aug2019.pdf?MOD=AJPERES. Accessed 24 June 2020.

9. Chu, S. K. W., H. Huang, W. N. M. Wong, W. F. van Ginneken, K. M. Wu, and M. Y. Hung. 2018. Quality and clarity of health information on Q\&A sites. Libr. Inf. Sci. Res. 40:237-244.

10. Davis, F. D. 1993. User acceptance of information technology: system characteristics, user perceptions and behavioral impacts. Int J. Man. Mach. Stud. 38:475-487.
11. Elshahat, S., J. V. Woodside, and M. C. McKinley. 2019. Meat thermometer usage amongst European and North American consumers: a scoping review. Food Control 106:106692.

12. Ferk, C. C., B. L. Calder, and M. E. Camire. 2016. Assessing the food safety knowledge of University of Maine students. J. Food Sci. Educ. 15:14-22.

13. Francis, J., M. P. Eccles, M. Johnston, A. E. Walker, J. M. Grimshaw, R. Foy, E. F. S. Kaner, L. Smith, and D. Bonetti. 2004. Constructing questionnaires based on the theory of planned behavior: a manual for health services researchers. Available at: https://openaccess.city.ac.uk/id/ eprint/1735/. Accessed 24 June 2020.

14. Green, E. J., and P. L. Knechtges. 2015. Food safety knowledge and practices of young adults. J. Environ. Health 77:18-24.

15. Havelaar, A. H., M. D. Kirk, P. R. Torgerson, H. J. Gibb, T. Hald, R. J. Lake, N. Praet, D. C. Bellinger, N. R. de Silva, N. Gargouri, N. Speybroeck, A. Cawthorne, C. Mathers, C. Stein, F. J. Angulo, and B. Devleesschauwer, on behalf of W.H.O. Foodborne Disease Burden Epidemiology Reference Group. 2015. World Health Organization global estimates and regional comparisons of the burden of foodborne disease in 2010. PLOS Med. 12:e1001923.

16. Henley, S. C., J. Gleason, and J. J. Quinlan. 2016. Don't wash your chicken!: A food safety education campaign to address a common food mishandling practice. Food Prot. Trends 36:43-53.

17. Henley, S. C., S. E. Stein, and J. J. Quinlan. 2015. Characterization of raw egg and poultry handling practices among minority consumers: identification of unique practices. Br. Food J. 117:3064-3075.

18. Koppel, K., F. Higa, S. Godwin, N. Gutierrez, R. Shalimov, P. Cardinal, B. Di Donfrancesco, M. Sosa, A. A. Carbonell-Barrachina, L. Timberg, and E. Chambers. 2016. Food leftover practices among consumers in selected countries in Europe, South and North America. Foods 5:66.
19. Kosa, K. M., S. C. Cates, S. Bradley, E. Chambers IV, and S. Godwin. 2015. Consumer-reported handling of raw poultry products at home: results from a national survey. J. Food Prot. 78:180-186.

20. Kosa, K. M., S. C. Cates, J. Brophy, S. Godwin, D. Chambers, and E. Chambers. 2019. Older adults and parents of young children have different handling practices for raw poultry. J. Food Prot. 82:200-206.

21. Lando, A. M., and C. C. Chen. 2012. Trends in ownership and usage of food thermometers in the United States, 1998 through 2010. J. Food Prot. 75:556-562.

22. Lukacsovics, A., A. Nesbitt, B. Marshall, R. Asplin, J. Stone, G. Embree, M. Hurst, and F. Pollari. 2014. Using environmental health officers' opinions to inform the source attribution of enteric disease: further analysis of the "most likely source of infection." BMC Publ. Health14:1258.

23. Ma, J., B. Almanza, R. Ghiselli, M. Vorvoreanu, and S. Sydnor. 2017. Food safety information on the Internet: consumer media preferences. Food Prot. Trends 37:247-255.

24. Mari, S., B. Tiozzo, D. Capozza, and L. Ravarotto. 2012. Are you cooking your meat enough? The efficacy of the theory of planned behavior in predicting a best practice to prevent salmonellosis. Food Res. Int. 45:1175-1183.

25. Marton, C., and C. Wei Choo. 2012. A review of theoretical models of health information seeking on the web. J. Doc. 68:330-352.

26. McLinden, T., J. M. Sargeant, M. K. Thomas, A. Papadopoulos, and A. Fazil. 2014. Component costs of foodborne illness: a scoping review. BMC Publ. Health 14:509.

27. Mullan, B., V. Allom, K. Sainsbury, and L. A. Monds. 2015. Examining the predictive utility of an extended theory of planned behavior model in the context of specific individual safe food-handling. Appetite 90:91-98.

28. Mullan, B. A., and C. L. Wong. 2009. Hygienic food handling behaviors. An application of the theory of planned behavior. Appetite 52:757-761. 
29. Murray, R., S. Glass-Kaastra, C. Gardhouse, B. Marshall, N. Ciampa, K. Franklin, M. Hurst, M. K. Thomas, and A. Nesbitt. 2017. Canadian consumer food safety practices and knowledge: foodbook study. J. Food Prot. 80:1711-1718.

30. Olsen, N. V., E. Røssvoll, S. Langsrud, and J. Scholderer. 2014. Hamburger hazards and emotions. Appetite 78:95-101.

31. Overbey, K. N., L.-A. Jaykus, and B. J. Chapman. 2017. A systematic review of the use of social media for food safety risk communication. J. Food Prot. 80:1537-1549.

32. Peterson, C. H., N. A. Peterson, and K. G. Powell. 2017. Cognitive interviewing for item development: validity evidence based on content and response processes. Meas. Eval. Couns. Dev. 50:217-223.

33. Record, R. A., W. R. Silberman, J. E. Santiago, and T. Ham. 2018. I sought it, I Reddit: examining health information engagement behaviors among Reddit users. J. Health Commun. 23:470-476.
34. U.S. Food and Drug Administration. 2017 2016 FDA food safety survey. Available at https://www.fda.gov/food/cfsan-consumerbehavior-research/2016-food-safety-surveyreport. Accessed 24 June 2020.

35. Vrbova, L., K. Johnson, Y. Whitfield, and D. Middleton. 2012. A descriptive study of reportable gastrointestinal illnesses in Ontario, Canada, from 2007 to 2009. BMC Publ. Health 12:970.

36. Yarrow, L., V. M. Remig, and M. M. Higgins 2008. Food safety awareness: concerns, practices, and openness to change of college students with health and non-health majors. Food Prot. Trends 28:585-591.

37. Young, I., M. Bhulabhai, and A. Papadopoulos. 2020. Safe food handling advice provided on question-and-answer websites is inconsistent. J. Nutr. Educ. Behav. 52:688-696.
38. Young, I., D. Reimer, J. Greig, P. Turgeon, R. Meldrum, and L. Waddell. 2017.

Psychosocial and health-status determinants of safe food handling among consumers: a systematic review and meta-analysis. Food Control 78:401-411.

39. Young, I., and L. Waddell. 2016. Barriers and facilitators to safe food handling among consumers: a systematic review and thematic synthesis of qualitative research studies. PLoS One 11:e0167695

40. Yun, E. K., and H.-A. Park. 2010. Consumers' disease information-seeking behavior on the Internet in Korea. J. Clin. Nurs. 19:2860-2868.

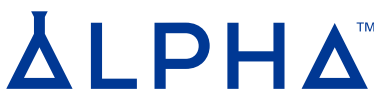

B I O S C I E N C E S

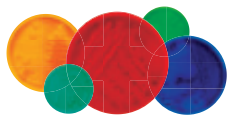

NOBODY

GROWS

BUGS

BETTER

SINCE 1999

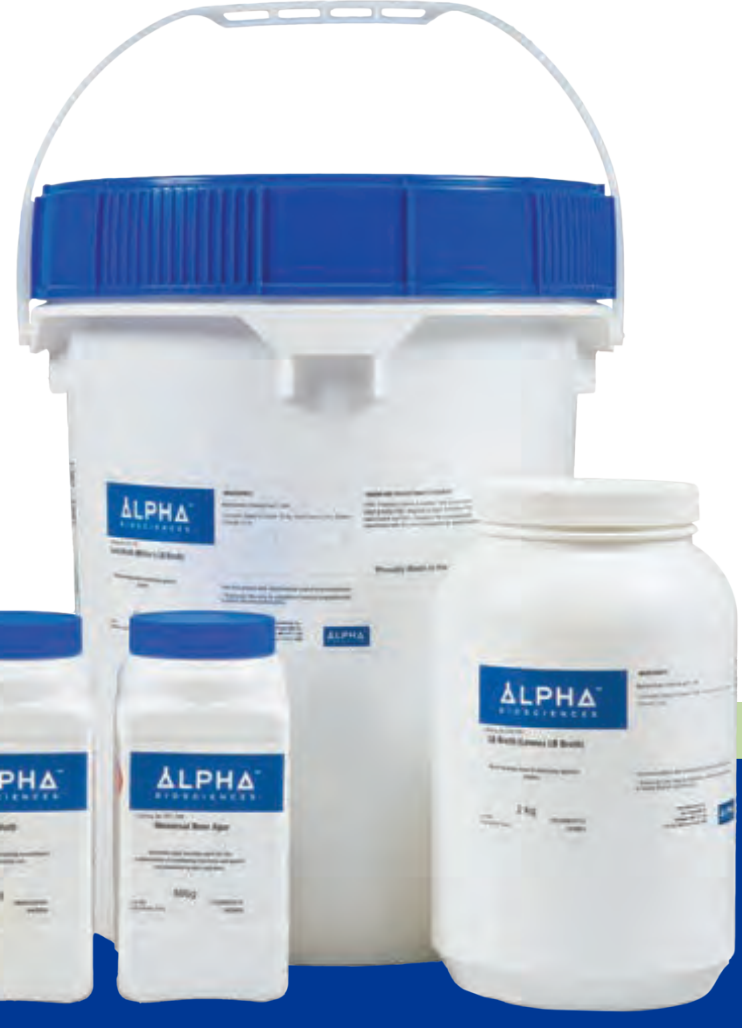

SORRY WE MISSED YOU AT IAFP 2020!

\section{Get your $\mathbf{2 5 \%}$ Off New Customer Discount Today. Use Code BUGS25.}

